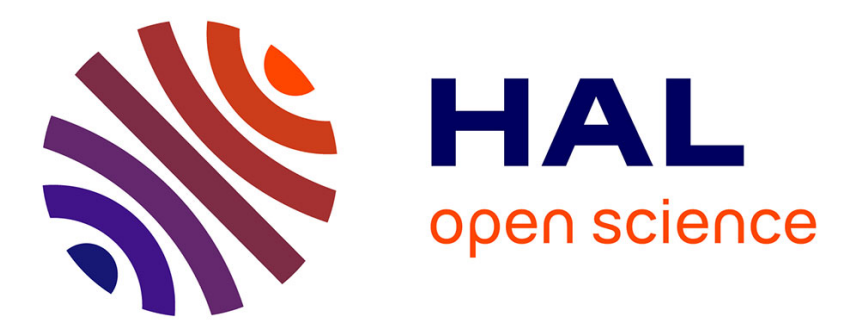

\title{
Écosystèmes audibles et structures sonores émergentes dans la musique d'Agostino Di Scipio. Une collaboration entre philosophie de la musique et analyse musicale
} Renaud Meric, Makis Solomos

\section{- To cite this version:}

Renaud Meric, Makis Solomos. Écosystèmes audibles et structures sonores émergentes dans la musique d'Agostino Di Scipio. Une collaboration entre philosophie de la musique et analyse musicale. Analyse Musicale, 2011, p. 39-56. hal-00770099

\section{HAL Id: hal-00770099 \\ https://hal.science/hal-00770099}

Submitted on 7 Jan 2013

HAL is a multi-disciplinary open access archive for the deposit and dissemination of scientific research documents, whether they are published or not. The documents may come from teaching and research institutions in France or abroad, or from public or private research centers.
L'archive ouverte pluridisciplinaire HAL, est destinée au dépôt et à la diffusion de documents scientifiques de niveau recherche, publiés ou non, émanant des établissements d'enseignement et de recherche français ou étrangers, des laboratoires publics ou privés. 


\title{
Écosystèmes audibles et structures sonores émergentes dans la musique d'Agostino Di Scipio. Une collaboration entre philosophie de la musique et analyse musicale
}

\author{
Renaud MERIC, Makis SOLOMOS*
}

\section{Structures sonores émergentes et écosystèmes audibles dans la musique d'Agostino Di Scipio}

Le compositeur italien Agostino Di Scipio (né en 1962) est connu pour ses œuvres utilisant l'électronique ou l'électronique et des instruments ou encore pour ses installations sonores ${ }^{1}$. Dans ces œuvres, il explore des idées théoriques nouvelles qu'il a fréquemment développées dans des $\operatorname{articles}^{2}$, idées qui ont parfois été

\footnotetext{
* Renaud Meric est Docteur en musicologie, Rirra21, Université Paul Valéry-Montpellier 3 ; Makis Solomos est Professeur de musicologie, Université Paris 8 . Une première version de ce texte est paru en anglais : "Audible Ecosystems and emergent sound structures in Di Scipio's music. Music philosophy helps musical analysis », Journal for Interdisciplinary Music Studies 3/1-2 (2009), p. 57-76.

1 Agostino DI SCIPIO, CD Hörbare Ökosysteme, live-elektronische Kompositionen 1993-2005, Berlin, Edition RZ, 2005 ; CD Paysages historiques. Musique électroacoustique 1998-2005, Bourges, Chrysopée électronique-Bourges, LDC 27811 30, 2005.

${ }^{2}$ Cf. par exemple Agostino DI SCIPIO, «Formal Processes of Timbre Composition. Challenging the Dualistic Paradigm of Computer Music », Proceedings of the 1994 International Computer Music Conference, San Francisco, International Computer Music Association, 1994, p. 202-208 ; «Synthesis of environmental sound textures by iterated nonlinear functions ", Proceedings of the $2^{\text {nd }}$ COST G-6 Workshop on Digital Audio Effects, Trondheim, NTNU, 1999; "Sound is the interface: from interactive to ecosystemic signal processing », Organised Sound 8/3, 2003, p. 269-277; «Due di Uno. A Composition Dedicated to Horacio Vaggione», Espaces composables. Essais sur la musique et la pensée musicale d'Horacio Vaggione, M. Solomos éd., Paris, L'Harmattan, 2007 ; «L'émergence du son, le son de l'émergence ", Intellectica. Revue de l'Association pour la Recherche Cognitive 48-49 (2008). Cf. aussi Christine ANDERSON, « Dynamical Networks of Sonic Interactions. An Interview with Agostino Di Scipio », Computer Music Journal 29/3 (2005) p. 11-28.
} 
débattues par des musicologues ${ }^{3}$. Dans le présent article, nous aimerions débattre de la question suivante : comment l'analyse musicale pourrait-elle traiter des œuvres musicales de Di Scipio ? Pour y répondre, nous verrons qu'il sera nécessaire de convoquer la philosophie de la musique : elle constituera un guide pour l'analyse. Mais avant d'en arriver à ce stade, résumons brièvement les idées de Di Scipio à l'aide de ses deux concepts les plus importants.

\section{Structures sonores émergentes}

Commentant l'hypothèse de Xenakis ${ }^{4}$ d'une (auto-)création de «sonorités de second ordre » dans l'œuvre de ce dernier intitulée Analogique A et B (1958-59, pour neuf cordes et bande) - une œuvre musicale qui utilise le paradigme granulaire -, Di Scipio décale légèrement la problématique de Xenakis : "Aujourd'hui, les sciences cognitives et l'épistémologie décriraient probablement l'hypothèse de sonorités de second ordre comme une question de propriétés émergentes de la structure sonore », écrit-il ${ }^{5}$. Ainsi, peut être formulée la question des structures émergentes : «Dans ce cas [à propos d'Analogique B], la distinction entre un modèle d'articulation et un modèle de son composé [sound design] peut difficilement se faire, dans la mesure où l'action du compositeur revient à laisser la structure musicale (niveau macro) émerger du son lui-même et de son organisation interne (niveau micro) $»^{6}$. Pour analyser l' «échec » d'Analogique $A$ et $B$ dans la recherche de sonorités de second ordre - qui n'est donc pas l'échec de la fusion des grains comme le pensait sans doute Xenakis, mais celui de l'émergence en question -, Di Scipio pointe le mode de construction: la stochastique. «On pourrait se demander si la stochastique fournit véritablement de bons moyens pour que des sonorités d'ordre supérieur émergent du comportement basique [ground-level pattern] d'unités sonores minimales $»^{7}$. Aussi, pour sa propre musique, il fera appel aux systèmes dynamiques complexes: «Le chaos et la dynamique des systèmes complexes, tels que rendus accessibles par des processus numériques itératifs, représentèrent pour moi une manière de composer de petites unités sonores de telle sorte qu'une sonorité d'ordre supérieur puisse se manifester d'elle-même durant le processus $»^{8}$. Ces systèmes lui permettent $«\left[\mathrm{~d}^{\prime}\right]$ exploiter $[\ldots$ une large $]$ palette

\footnotetext{
${ }^{3}$ Cf. Makis Solomos, « Notes sur Agostino Di Scipio et sur la notion d'émergence », $12^{e}$ Journées d'Informatique Musicale 2005, A. Sedes et H. Vaggione éd., Paris, Université Paris 8 - CICM - MSH Paris Nord - Afim, 2005, p. 101-109 ; M. PROMINSKI, « Ökosysteme Entwerfen », http://www.ila.unihannover.de/fileadmin/theorie_landschaftsarchitektur/documents ; Renaud MERIC, «Le bruit de fond est-il un son? À propos d'Écosystèmes audibles $3 a$ d'Agostino Di Scipio », Musique et bruit. Filigrane 7 (2008), p. 197-213.

${ }^{4}$ Cf. Iannis XenAKIS, Formalized Music, translated and edited by Sh. Kanach. Stuyvesant, New York, Pendragon Press, 1992, p. 103.

${ }^{5}$ Agostino DI SCIPIO, «Clarification on Xenakis: the Cybernetics of Stochastic Music », Présences de / Presences of Iannis Xenakis, M. Solomos éd., Paris, Cdmc, 2001, p. 71-84, p. 72.

6 Agostino DI SCIPIO, «The problem of $2^{\text {nd }}$-order sonorites in Xenakis' electroacoustic music », Organised Sound 2/3 (1997), p. 165.

${ }_{7}^{7}$ Agostino Di SCIPIO, « Clarification on Xenakis... », op. cit., p. 73 et 79.

${ }^{8}$ Agostino Di SCIPIO, cité par C. Anderson, op. cit.
} 
d'arrangements de grains, allant de textures aléatoires à des textures aux motifs plus déterminés [more patterned textures], en passant par une variété d'autres comportements »". Aussi, Di Scipio, à la différence d'autres compositeurs qui ont utilisé le paradigme granulaire - par exemple, Xenakis ou Horacio Vaggione ${ }^{10}-$ tend à se débarrasser de tout ce qui serait composé à l'échelle de la macroforme. Par exemple, dans sa musique, il n'y a ni gestes ni intentions dramatiques. Dans un de ses premiers articles ${ }^{11}$, il élabora une «théorie de l'émergence sonologique» (Theory of sonological emergence), où la forme est conçue comme «formation du timbre ».

L'idée de structures sonores émergentes est liée à l'élaboration d'une stratégie musicale sub-symbolique. Avec la théorie de l'émergence sonologique, Di Scipio escompte l'émergence d'un niveau supérieur en travaillant précisément sur des grains, des échantillons, des éléments qui ne constituent pas des symboles, car ils se situent à un niveau inférieur ${ }^{12}$. Avec les interactions composées (cf. infra), il situe l'interaction directement au niveau du signal sonore: tous les échanges d'information sont de nature purement sonore ${ }^{13}$. Nous pouvons établir un parallèle entre cette stratégie musicale et le modèle de l'émergence dans les sciences cognitives. À la question «Qu'est-ce que la cognition?», le computationnalisme répond : «Le traitement de l'information : la manipulation de symboles à partir de règles ${ }^{14}$, alors que le modèle de l'émergence répond: "L'émergence d'états globaux dans un réseau de composants simples ${ }^{15}$. Au niveau musical, l'enjeu est le suivant: si l'on veut que le niveau supérieur, celui de la macroforme, se présente comme une émergence et non comme une construction indépendante, on ne doit travailler que sur le niveau inférieur, délaissant le niveau intermédiaire constitué de symboles.

\section{Écosystèmes audibles}

Selon la théorie de l'émergence sonologique, l'émergence de structures sonores est possible grâce au fait que le compositeur développe des systèmes (au sens de la cybernétique) proches des systèmes vivants, qui sont caractérisés par une capacité d'auto-organisation: «Le passage d'un système ou d'un processus d'une organisation structurelle donnée à un nouvel état d'ordre reconnu comme fonction des propriétés qualitatives de celle-ci est ce que nous nommons ici un phénomène d'émergence $[\ldots]$. Des phénomènes similaires peuvent être décrits selon des règles

\footnotetext{
${ }^{9}$ Ibid.

${ }^{10}$ Cf. Makis Solomos, « The granular connection (Xenakis, Vaggione, Di Scipio...) », communication au colloque The Creative and Scientific Legacies of Iannis Xenakis International Symposium, organisé par J. Harley, M. Duschenes, T. Salisbury, University of Guelph / Perimeter Institute for Theoretical Physics / Fields Institute for Research in Mathematical Sciences (Canada).

${ }^{11}$ Agostino DI SCIPIO, «Formal Processes... », op. cit., p. 205.

${ }^{12}$ Ibid., p. 203.

${ }_{13}^{13}$ Agostino DI SCIPIO, « Sound is the interface... », op. cit., p. 272.

${ }^{14}$ Francesco VARELA, Invitation aux sciences cognitives, Paris, Seuil, 1996, p. 42.

${ }^{15}$ Ibid., p. 77.
} 
de morphostase (conservation de la cohérence, de l'identité) ou de morphogenèse (comportement dynamique, changement), qui, ensemble, saisissent la particularité principale de systèmes sociaux et vivants : l'auto-organisation ${ }^{16}$. Pour s'assurer de l'auto-organisation dans un domaine où cette idée ne va pas de soi - le son ou la musique ne sont pas «vivants »-, Di Scipio pratique une «causalité circulaire ${ }^{17}$ (ou récursivité), qui élargit la notion de feedback. Par exemple, dans Due di Uno (2003, pour violon, piccolo, flûte à bec et DSP adaptatif), les sons instrumentaux, qui sont transformés par l'électronique, servent aussi d'entrée pour contrôler ces transformations ${ }^{18}$.

Grâce à cette causalité circulaire, Di Scipio redéfinit la notion usuelle, en musique avec dispositif électronique en direct, de la notion d'interaction ${ }^{19}$. Dans le modèle le plus commun de l'interaction en direct, l'interaction est surtout comprise comme un flux d'information : une source sonore est transformée. En quelque sorte, le système agent + dispositif est peu interactif. Selon Di Scipio, la composition ellemême pourrait devenir le résultat du fait de composer des interactions. Ainsi, « un objectif primordial serait de créer un système dynamique possédant un comportement adaptatif aux conditions extérieures environnantes, et capable d'interagir avec les conditions extérieures elles-mêmes. [...] Une sorte d'auto-organisation est ainsi accomplie [...] Ici, l' "interaction" est un élément structurel pour que quelque chose comme un "système" puisse émerger [...] Les interactions du système seraient alors implémentées seulement d'une manière indirecte, elles seraient les produits secondaires d'interdépendances soigneusement planifiées entre les composants du système $[\ldots]$. On s'écarte ainsi d'une manière substantielle de la composition musicale interactive pour aller vers la composition d'interactions musicales ; peutêtre pourrions-nous parler, pour être plus précis, du passage de la création de sons voulus selon des moyens interactifs à la création d'interactions voulues laissant des traces audibles $\gg{ }^{20}$. Nous pourrions dire que, pour Di Scipio, la notion de processus est décisive : le processus est plus important que le résultat - et que l'origine.

Cependant, l'interaction a également lieu avec l'environnement acoustique : un autre élément important de l'approche de Di Scipio est l'idée d'écosystème. Dans l'ensemble de pièces intitulées Audible Ecosystemics (2002-2005, pour solo d'électronique en direct) - ensemble qui offre l'accomplissement musical d'interactions composées) -, l'écosystème est formé par une interaction triangulaire entre le musicien, les patches DSP et l'ambiance sonore (cf. exemple 1$)^{21}$. Ceci entrâne un rôle particulier accordé au bruit. Pour simplifier, on dira que le bruit n'est plus perturbation (musique traditionnelle) ou nouveau matériau à transformer (musique contemporaine). Il devient l'un des agents de l'interaction, puisqu'il émane de l'espace concret - le lieu, l'environnement -, qui fait intégralement partie

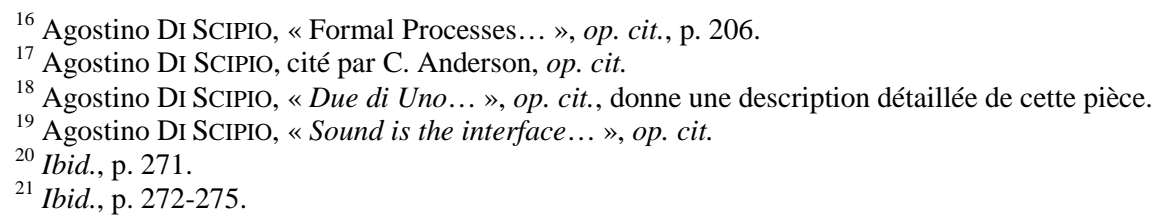


du système. Dans les Audible Ecosystems, «le rôle du bruit est crucial [...]. Le bruit est le milieu [medium] lui-même où un système à génération sonore est situé, il constitue, strictement parlant, son ambiance. En outre, le bruit est l'énergie fournie grâce à laquelle un système auto-organisé peut se maintenir et se développer $»^{22}$.

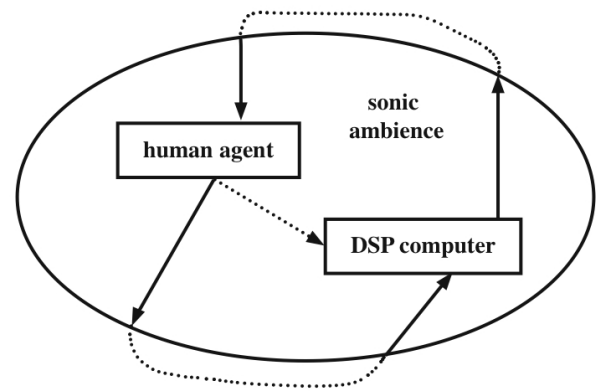

Triangular recursive ecosystemic connection.

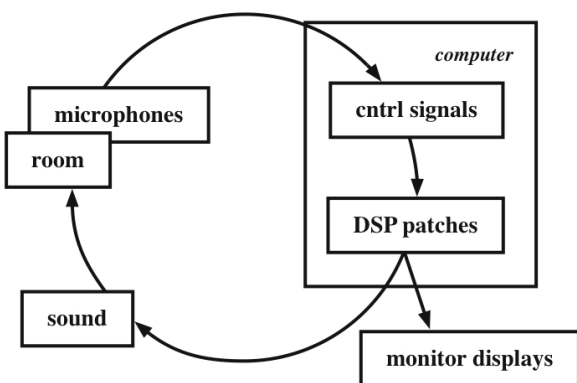

Basic design of the Audible Eco-Systemic Interface.

Exemple 1. Interactions composées pour l'Interface audible écosystémique ${ }^{23}$.

La première cherche à savoir comment la musique a été composée. Cette recherche peut être liée à des propos biographiques, psychanalytiques, culturels, esthétiques, etc. ; dans ce cas, nous ne sommes pas à proprement parler dans les cadres de l'analyse. Elle peut être liée à des études sur les esquisses ou sur les différentes versions de l'œuvre; dans ce cas, l'ancienne discipline de l'analyse se renouvelle sous l'appellation de critique "génétique». Avec ce type de travaux, l'analyse se concentre sur des aspects philologiques ou bien - si elle combine cette approche avec la question des «règles » déterminant l'œuvre - elle cherche à trouver - ou à (ré)inventer - le processus générateur de l'œuvre musicale.

${ }^{22}$ Ibid., p. 271

${ }^{23}$ Agostino Di SCIPIO, « Sound is the interface ... », op. cit., p. 272. 
La seconde catégorie aborde l'œuvre au niveau de la musique «elle-même ». Elle part du « résultat » (le résultat du processus générateur), c'est-à-dire de l'œuvre musicale «elle-même ». Suivant cette approche, l'analyste pense que « les "règles" donnés par un compositeur ne prouvent rien à propos de la consistance de la musique, au contraire de la situation du scientifique pour qui les règles (qui sont comprises, sinon partagées, collectivement) constituent la seule chose qu'il peut montrer pour légitimer son œuvre $»^{24} 25$. Aussi, il traite de la musique en tant que monde déterminé et fini. Selon cette manière de penser l'analyse, ce que nous entendons est tout aussi important - ou même plus - que ce qui se trouve «sous » les structures perçues ou que les intentions compositionnelles. L'analyste part de l'hypothèse que la musique (l'œuvre musicale à analyser) constitue une entité, qui est fixée, qui est là, prête à être analysée en tant que construction. Dans le cas de la musique électroacoustique, le résultat - le «monde », l'« univers », la construction, qui se trouve «là »- est le «son »: il s'agit d'un monde sonore, d'une entité sonore, d'une construction sonore. Dans le champ de la musique électroacoustique, il existe une tradition analytique importante qui part de cette approche, et qui se fonde sur la notion schaefférienne d' « objet sonore », sur l'analyse spectromorphologique de Denis Smalley, sur des méthodes inspirées par la phonologie, sur les méthodes «paradigmatique », « fonctionnelle », « générative » ou «implicative ${ }^{26}$, ou encore sur des méthodes plus ou moins empiriques, etc. Quelquefois, le propos est déterminé par la question de la visualisation du son, et l'analyse est constituée par des transcriptions visuelles - comme dans le cas du logiciel Acousmographe du GRM - mettant en évidence les articulations ou tout autre élément méta-sonore.

Dans notre recherche sur Agostino Di Scipio, notre propos n'est pas de trouver le processus générateur de sa musique, ni d'étudier des détails philologiques. Nous ne cherchons pas à savoir comment la musique a été composée. Il reste donc le choix du second type d'approche. Mais qu'est-ce que la musique elle-même? Qu'est-ce que le résultat? - nous avons vu que Di Scipio ne pense pas la musique comme un «résultat». Plus précisément, qu'est-ce qu'un résultat sonore?

\footnotetext{
${ }^{24}$ Horacio VAGgione, «Analysis and the Singularity of Music: The Locus of an Intersection », Analyse en Musique Électroacoustique / Analysis in Electroacoustic Music, Académie Internationale de Musique de Bourges, vol. II, Bourges, Mnémosyne, 1997, p. 270.

${ }^{25}$ Le cas du sérialisme est typique de cette situation, et c'est pourquoi Boulez lui-même dit que, pour analyser la musique sérielle, il part directement du résultat : «Est-ce bien nécessaire d'analyser les œuvres à partir de leurs procédures? Je pense qu'il est préférable de les analyser en tant que résultats. Je me suis moi-même très vite aperçu en analysant Webern à partir du comptage des notes de la série que cela ne me donnait pas de clé. Ce qui m'intéresse par exemple dans le premier mouvement de la Symphonie opus 21, ce n'est pas de voir que les douze sons ont été respectés, ou qu'il s'agit d'un canon, mais pourquoi on n'entend pas le canon, pourquoi il disparaît sous la sensation harmonique » («Entretien avec Pierre Boulez », Pli selon pli de Boulez, Genève, Contrechamps, 2003, p. 10). Pour une proposition analytique sur la Symphonie op. 21 de Webern par-delà les analyses des structures compositionnelles, cf. Makis Solomos, «Le premier mouvement de la Symphonie op. 21 de Webern ", Les Cahiers du CIREM 42-43 (1998), p. 117-124.

${ }^{26}$ Les quatre dernières méthodes sont utilisées par Stéphane ROY, L'analyse des musiques électroacoustiques : Modèles et propositions, Paris, L'Harmattan, 2003.
} 


\section{Une image analytique}

Pour Agostino Di Scipio, nous l'avons vu, le processus est plus important que le résultat. Cette idée pose problème lorsque nous tentons d'analyser sa musique. Chercher un résultat - un résultat sonore - en l'analysant et en tentant de le délimiter peut être un non-sens pour sa musique. Pour les Audible ecosystemics, le seul résultat auquel nous pouvons être confrontés est une écoute subjective et éphémère. Ce que j'ai «entendu » ne peut pas réapparaître. Par cette affirmation, nous ne revendiquons pas ici une pure idée solipsiste : nous prenons tout simplement en considération les spécificités émergentes de ces œuvres musicales. Dans les Audible ecosystemics, l'écosystème n'est pas uniquement une interaction triangulaire entre le musicien, l'ordinateur (le système de traitement du signal numérique) et une ambiance sonore ; il exige également une interaction étroite entre l'œuvre et l'auditeur. Tous deux sont des composants de l'écosystème :

Les auditeurs [listeners] sont un genre très particulier d'observateur ou d'écouteur [hearer] externes car leur simple présence physique dans la salle agit comme un élément d'absorption acoustique. Ils constituent donc plutôt un composant interne aux dynamiques de l'écosystème. On le sait, des répétitions sans public sont loin de répondre au contexte de la représentation réelle, et même un public relativement restreint modifie profondément la réponse de la salle. Pour le projet AESI [Audible Eco-Systemic Interface], ceci n'est considéré ni comme un problème, ni comme un élément extérieur à la musique : les changements dans l'ambiance révéleront des changements particuliers dans l'ensemble des dynamiques de l'écosystème, et donc dans les résultats audibles euxmêmes ${ }^{27}$.

L'auditeur et l'œuvre se trouvent dans le même espace et dans le même temps : il est impossible de délimiter un espace et un temps propre à la musique ${ }^{28}$. Cette remarque est cruciale dans la mesure où la première étape d'une analyse musicale consiste généralement à effectuer une telle démarcation. En d'autres termes, l'analyste commence par choisir un point de vue en délimitant ce qu'il considère comme objectif et ce qu'il considère comme subjectif. L'œuvre acquiert alors un espace et temps spécifiques, comme si elle était un univers distinct, une entité avec sa propre structure.

De ce point de vue, l'œuvre n'est rien d'autre qu'une image. L'analyse musicale d'une œuvre peut ainsi être considérée comme une analyse d'image. L'analyse utilise différents outils pour décrire, "disséquer», schématiser cette "image». L'analyste n'observe pas directement l'œuvre musicale ou ce qu'il écoute, mais l'image qu'il a établie à partir d'elle. Qu'est-ce que cela induit? Lorsque l'analyste transpose l'œuvre musicale en une image, il conçoit une entité statique et universelle. L'opposition entre la «vision syncrétique » et la «vision analytique » dessinée par Anton Ehrenzweig pourrait expliquer cette situation :

\footnotetext{
${ }^{27}$ Agostino Di SCIPIO, « Sound is the interface... », op. cit., p. 274.

${ }^{28}$ Cf. Renaud MERIC, «Le bruit de fond est-il un son? », op. cit.
} 
La structure indifférenciée de la fantasmatique du processus primaire correspond à la structure primitive encore indifférenciée de la vision chez l'enfant. Pour désigner cette qualité particulière de la vision de l'enfant, Piaget a répandu le terme de vision «syncrétique ». Or, le syncrétisme implique le concept d'indifférenciation. Autour de la huitième année, l'art enfantin subit un changement radical, du moins dans la civilisation occidentale. Tandis que le jeune enfant fait des expériences audacieuses de forme et de couleur en représentant toutes sortes d'objets, l'enfant plus âgé commence à analyser ces formes en les confrontant avec l'art adulte qu'il trouve dans les magazines, les livres et les images. [...]. Ce qui est arrivé, c'est que la vision de l'enfant a cessé d'être totale et syncrétique, pour devenir analytique. La vision syncrétique, plus primitive de l'enfant, ne différencie pas, comme celle d'un adulte, les détails abstraits. L'enfant ne décompose pas la forme d'un objet concret en éléments abstraits plus petits, pour ensuite confronter un à un les éléments de ses dessins. Sa vision est encore globale et comprend tout l'ensemble qui reste indifférencié quant aux détails qui le composent ${ }^{29}$.

La vision analytique doit être statique. Chaque élément, chaque détail doit avoir une place spécifique dans le temps et dans l'espace ou, plus précisément, dans le temps et dans l'espace propre à une image. Les mouvements ou les transformations n'ont alors plus lieu d'être. Inversement, une vision syncrétique implique des mouvements continus, sans limites particulières.

\section{Empreinte}

Il est très difficile d'avoir une perception analytique de la musique d'Agostino Di Scipio. Rien dans sa musique ne peut être considéré comme statique. Les Audible ecosystemics ne mettent en œuvre aucun son spécifique préétabli - aucun instrument, aucun échantillon, aucun enregistrement. Par exemple, l'Audible ecosystemics 3a - Background noise study «débute avec «rien» (nous ne prêtons habituellement pas attention aux évènements sonores à peine audibles; inconsciemment, nous les scotomisons) et tente de construire quelque chose à partir de lui $»^{30}$. Pour l'Audible ecosystemics $3 \mathrm{~b}-$ Background noise study, with mouth performer, « tous les petits sons involontairement produits dans la bouche et dans la gorge constituent la source $»^{31}$. Tous ces sons sont imprévisibles et généralement inaudibles. Ils sont captés par deux microphones dans la salle (un seul dans la Background noise study $3 b$, with mouth performer, qui est placé dans la bouche ou près des lèvres), et ils sont envoyés - sous forme de signaux numériques - vers l'unité de traitement du signal numérique (système DSP). À l'intérieur de cette unité DSP, il n'y a aucun chemin linéaire ou direct: les signaux en entrée sont conduits vers différents canaux. Certains signaux passent à travers des blocs de traitement du signal audio et d'autres à travers des blocs établissant des signaux de

${ }^{29}$ Anton EhrenzWeIG, L'ordre caché de l'art, trad. F. Lacoue-Labarthe et C. Nancy, Paris, Gallimard, 1974, p. 40.

${ }^{30}$ Agostino DI SCIPIO, CD Hörbare Ökosysteme, live-elektronische Kompositionen 1993-2005, op. cit., livret, p. 20

${ }^{31}$ Ibid. 
contrôle. En analysant le réseau DSP pour les Audible ecosystemics, nous découvrons une multitude de boucles : lorsqu'il sort d'un bloc, le signal est envoyé vers plusieurs autres blocs - qui le traitent ou qui utilisent ses valeurs en tant que signaux de contrôle - et il peut ainsi suivre des chemins variés. Tôt ou tard, le signal d'origine - passé par plusieurs blocs - retournera vers l'un de ces blocs, formant ainsi une boucle (cf. exemple 2).

Chaque signal - chaque partie de ce signal - peut être considéré simultanément comme une donnée sonore et comme une donnée de traitement. En d'autres termes, le signal est à la fois le matériau et le geste qui déforme ce matériau. Ce processus a lieu lorsque le son émerge de l'un des huit haut-parleurs : ce son vient intensifier le bruit de fond (les haut-parleurs sont orientés vers les murs et à proximité de ces derniers), qui est immédiatement capté par les deux microphones. Nous revenons ainsi au point de départ : une boucle se forme. En conclusion, il n'y a ni fin, ni début de l'écosystème. Chaque élément peut-être à la fois la fin et le début de ce dernier :

Les écosystèmes sont des systèmes dont la structure et le développement ne peuvent pas exister (encore moins être observé ou modelé) sans un contact permanent avec un milieu. Ils sont autonomes (c'est-à-dire, littéralement, autorégulés) étant donné que leur processus reflète leur propre structure interne. Mais ils ne peuvent pas être isolés du monde extérieur, et ne peuvent accomplir leur propre fonction autonome s'ils ne sont pas en conjonction étroite avec une source d'information (ou d'énergie). Les séparer de leur milieu revient à les tuer ${ }^{32}$.

Chaque partie de l'écosystème - à l'intérieur ou à l'extérieur du système DSP est toujours une source d'énergie pour les autres parties, mais elle utilise aussi l'énergie traitée par ces autres parties. Dans cette structure fermée, l'auditeur peut être lui-même considéré comme un élément de cette structure, à l'instar d'un bloc dans le système DSP, avec une entrée (le bruit de fond qu'il écoute et qui émerge des haut-parleurs) et une sortie (les modifications qu'il crée dans ce bruit de fond, avant que ce dernier ne soit capté par les deux micros).

${ }^{32}$ Agostino DI SCIPIO, « Sound is the interface... », op. cit., p. 271. 


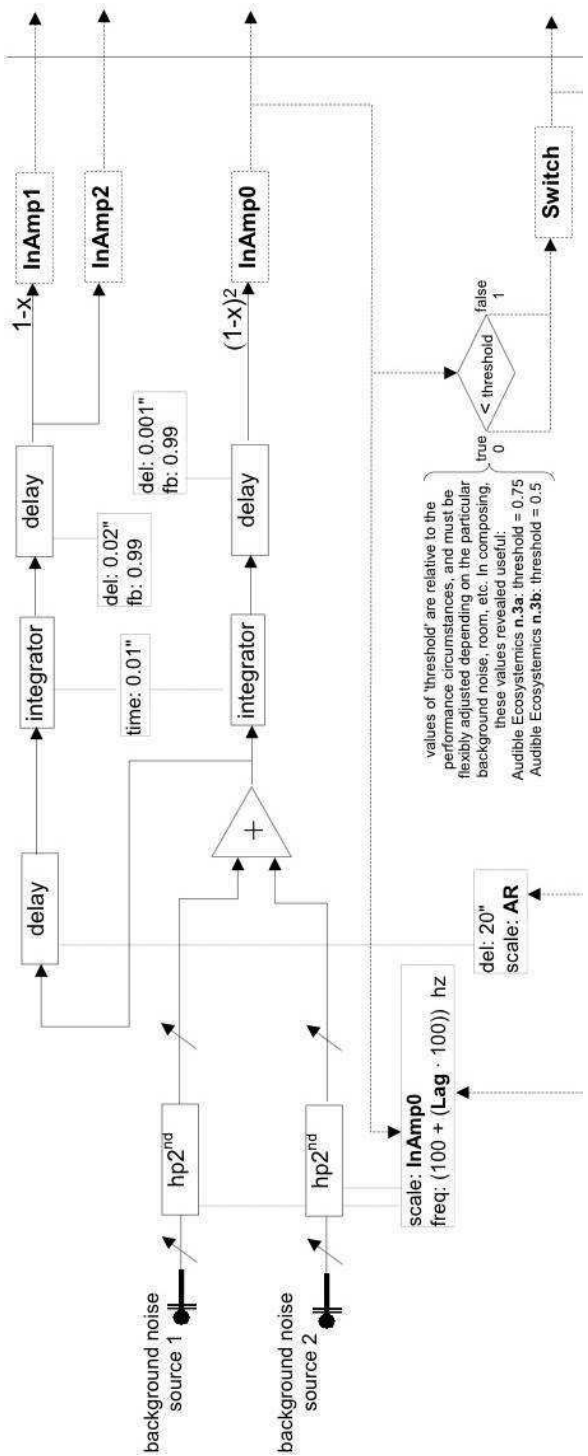

Exemple 2. Partie du schéma DSP pour les Audible ecosystemics $3 a / 3 b^{33}$. Sur cette figure, nous pouvons voir une boucle : [Hp2nd] $\rightarrow$ [integrator] $\rightarrow$ [delay] $\rightarrow$ [InAmp0] $\rightarrow$ [scale: InAmp0 / freq: (100+(lag.100))hz] $\rightarrow$ [Hp2nd].

${ }^{33}$ Agostino DI SCIPIO, Audible ecosystemics, $n^{\circ} 3 a /$ Background Noise Study $n^{\circ} 3 b /$ Background Noise study, with mouth performer(s) (Instructions and score), Berlin, Berliner Künstlerprogramme des DAAD, 2004-2005, p. 6, http://xoomer.alice.it/adiscipi/internal_backgroundnoisestudy.htm. 
Une telle description de la situation d'écoute peut sembler simpliste et caricaturale. Cependant, l' « entrée »du «bloc auditeur » correspond tout simplement à la réponse habituelle que nous donnons à la question «qu'est-ce nous écoutons ? : ce qui sort des haut-parleurs... L'identification traditionnelle de la musique avec ce qui sort des haut-parleurs semble ici inappropriée. Pourquoi ? Une analyse musicale qui se fonde sur la question «qu'est ce que nous écoutons? » recherche une image statique, une image avec sa propre structure (son propre espace et son propre temps). En procédant ainsi, l'analyste extirpe l'auditeur de l'écosystème et considère le son uniquement comme matériau sonore - comme le seul matériau audible. Or, nous avons observé qu'à chaque étape de l'écosystème, le son - le signal sonore - est toujours à la fois le matériau et le geste qui déforme ce dernier. Dans les Audible ecosystemics, ce que nous écoutons n'est pas strictement un son, mais plutôt la propre empreinte de ce dernier.

Afin de comprendre cette idée d'empreinte, nous devons faire référence au philosophe et à l'historien de l'art Georges Didi-Huberman. Dans son ouvrage $L a$ ressemblance par contact, Georges Didi-Huberman explique l'importance du travail de l'ichnologue (le paléontologue spécialiste des traces fossiles) pour le théoricien de l'art. Nous pourrions ainsi avancer un propos similaire pour la musique, en particulier pour comprendre la notion de structure musicale, et plus précisément dans le cas des structures écosystémiques d'Agostino Di Scipio :

[L'ichnologue] est obligé de reconnaître la complexité des formes, il est obligé de savoir que les formes sont des processus, et pas seulement le résultat de processus ; que ces processus, à proprement parler, n'ont pas de fin, que l'image actuellement vue n'est que le "présent anachronique" d'un jeu ininterrompu de déformations, d'altérations, d'effacements et de "revenances" de toutes sortes. D'autre part, l'ichnologue n'a nul besoin de questionner ce qu'il regarde comme l'iconographie d'un contenu ou l'expression d'un désir mimétique. La ressemblance offerte dans chaque empreinte est d'un tout autre ordre ; en elle rien ne peut être désintriqué, les formes de la matière par exemple. Car ici, les formes sont des substrats, ou plutôt le processus dialectique des modifications du substrat par un geste quelconque. L'ichnologue, enfin, n'a pas la naïveté de situer ce qu'il voit comme un point unique, intangible de l'histoire. [...]. Il sait donc que les formes sont des temps à l'œuvre, des temps contradictoires intriqués dans la même image : temps de la terre et temps du pied qui, un instant s'y est posé pour toujours ${ }^{34}$.

\section{Retour à l'analyse. Qu'est-ce qui se meut...}

Lorsque nous analysons une œuvre musicale comme une image statique, nous ne prenons pas en considération ce que Georges Didi-Huberman dit à propos des formes ( $\ll$ les formes sont des processus », et pas seulement le résultat de processus ; «les formes sont des substrats »; « les formes sont des temps à l'œuvre, des temps contradictoires intriqués dans la même image »). Lorsque nous explicitons

\footnotetext{
34 Georges DIDI-HuBERMAN, La ressemblance par contact; Archéologie, anachronisme et modernité de l'empreinte, Paris, Minuit, 2008, pp. 324-325.
} 
une structure dans une œuvre musicale, nous ne devons pas oublier qu'elle est un simple fragment d'espace et de temps. Dans les Audible ecosystemics, cette affirmation est plus qu'évidente : nous écoutons en même temps le processus et le son. Le son (que nous écoutons) est ici une simple étape dans un processus plus global et, par conséquent, d'une structure plus globale.

La question fondamentale de l'analyse musicale - qu'est ce que nous écoutons ? - montre ses propres limites, puisqu'elle aspire à une image statique avec un espace et un temps spécifiques. Dans les Audible ecosystemics, l'auditeur étant luimême une partie de l'œuvre écosystémique, il appartient au même temps, au même espace que l'œuvre, que le son. Nous devons poser la question d'une autre manière : qu'est-ce qui se meut dans ce que j'écoute? Lorsque nous cherchons ce qui se meut dans ce que nous écoutons, nous ne nous focalisons pas sur un résultat, mais sur un processus structurel. L'auditeur reste ainsi à l'intérieur de l'écosystème. Les mouvements ou les processus que nous écoutons sont une émergence éphémère de l'écosystème dans son ensemble. Ce qui émerge peut alors être considéré tout autant comme objectif que comme subjectif : il n'est pas la fin, l'extrémité finale, des interactions circulaires. Les mouvements que nous pouvons entendre, décrire et analyser constituent une toute petite partie des autres mouvements multiples que nous pouvons seulement imaginer. Par exemple, un simple petit «clic» que nous pouvons distinguer dans les Audible ecosystemics ne peut pas être considéré comme un objet distinct parmi les autres ou comme un détail à l'intérieur d'une image structurée. Il est la partie émergée de mouvements entrelacés qui s'affrontent et s'entrechoquent, qui n'émergent jamais et qui ne sont pas audibles. L'imagination, seule, peut atteindre cette partie inaudible de la structure écosystémique. Le «clic » entendu dans l'œuvre n'a aucun intérêt sans cette histoire, cette genèse, historique et inaudible. Chaque son constitue une empreinte mouvante d'une structure mouvante sous-jacente. Si nous commençons une analyse en nous posant la question «qu'est-ce qui se meut dans ce que j'écoute ? » au lieu de «qu'est ce que j'écoute? », nous cherchons à être totalement immergés dans la genèse du «clic ». Nous nous concentrons alors, non sur un objet - une partie bien délimitée de l'espace et du temps -, mais nous cherchons à comprendre pourquoi et comment un son particulier émerge simultanément dans la musique et dans l'imagination de l'auditeur.

Revenons à l'analyse : nous pourrions commencer une analyse en cherchant la genèse d'un simple son - en d'autres termes, d'un processus - que nous pouvons saisir dans l'un des Audible ecosystemics. Il peu s'agir de n'importe quel son, pris au hasard. Ce son émerge corrélativement d'un processus particulier et d'une écoute spécifique : autrement dit, d'un écosystème. Comme nous l'avons mentionné précédemment, nous ne devons pas considérer ce son comme une entité structurée statique et bien délimitée : il est en effet apparu une seule fois et il n'émergera plus jamais. Il n'est rien de plus qu'un cas singulier, un simple exemple.

Qu'est-ce qui se meut dans ce son particulier? Grâce à cette question, nous n'analysons pas seulement un son - un objet sonore - mais un phénomène mouvant. Ce simple son devient alors un phénomène complexe et hétéroclite, composé de multiples mouvements. Lorsque nous nous concentrons sur ce qui se meut, nous 
devons définir l'espace créé par ces mouvements à l'intérieur duquel ils émergent. Nous devons aussi questionner les relations entre ces divers mouvements. Nous interrogeons ainsi notre propre perception et notre propre imagination. Petit à petit et de mouvement en mouvement, le son étudié disparait en tant qu'entité : une structure complexe imaginaire se dessine alors. Dans cette structure complexe, l'écoute ne constitue pas le résultat, la finalité ou la fin de la structure musicale mais plutôt le début, le fondement de cette dernière. Dans les Audible ecosystemics d'Agostino Di Scipio, la structure est un phénomène sous-jacent qui est, à proprement parler, inaudible et invisible. La question «qu'est-ce qui se meut dans ce que j'écoute? » nous permet de révéler cette structure partiellement.

Cette question nous permet également d'aborder la composition musicale. Les réponses qu'elle induit - les mouvements que nous cherchons - concernent également les processus composés. Nous pouvons essayer de comprendre pourquoi et comment ce son particulier - ce processus - que nous désirons analyser émerge. L'étude des interactions composées ${ }^{35}$ nous aide à comprendre l'origine et la forme de ce son spécifique.

Finalement, une analyse musicale des Audible ecosystemics d'Agostino Di Scipio exige une analyse des relations entre ce que nous écoutons, ce que nous ne pouvons qu'imaginer et les techniques compositionnelles.

\section{Un son pris au hasard}

Cet article focalisera maintenant son analyse sur l'Audible Ecosystemics $3 b$. Background noise study, with mouth performer (2005), pour un performer, un microphone manipulé par le performer qui le place dans sa bouche, une unité DSP (soit le programme Kyma, soit le programme Pure Data) et 8 haut-parleurs orientés vers les murs. L'exemple 3 montre la partition destinée au performer (cette partition est suivie d'une série d'instructions que nous ne détaillons pas ici). Sans support audio, nous ne pouvons pas ici analyser un échantillon spécifique - par exemple, un échantillon bien délimité d'une seconde - extrait au hasard d'un enregistrement de cette œuvre. Nous tentons simplement de montrer l'intérêt d'une telle analyse.

\footnotetext{
${ }^{35}$ Voir la partition et le schéma du réseau DSP des Audible ecosystemics.
} 


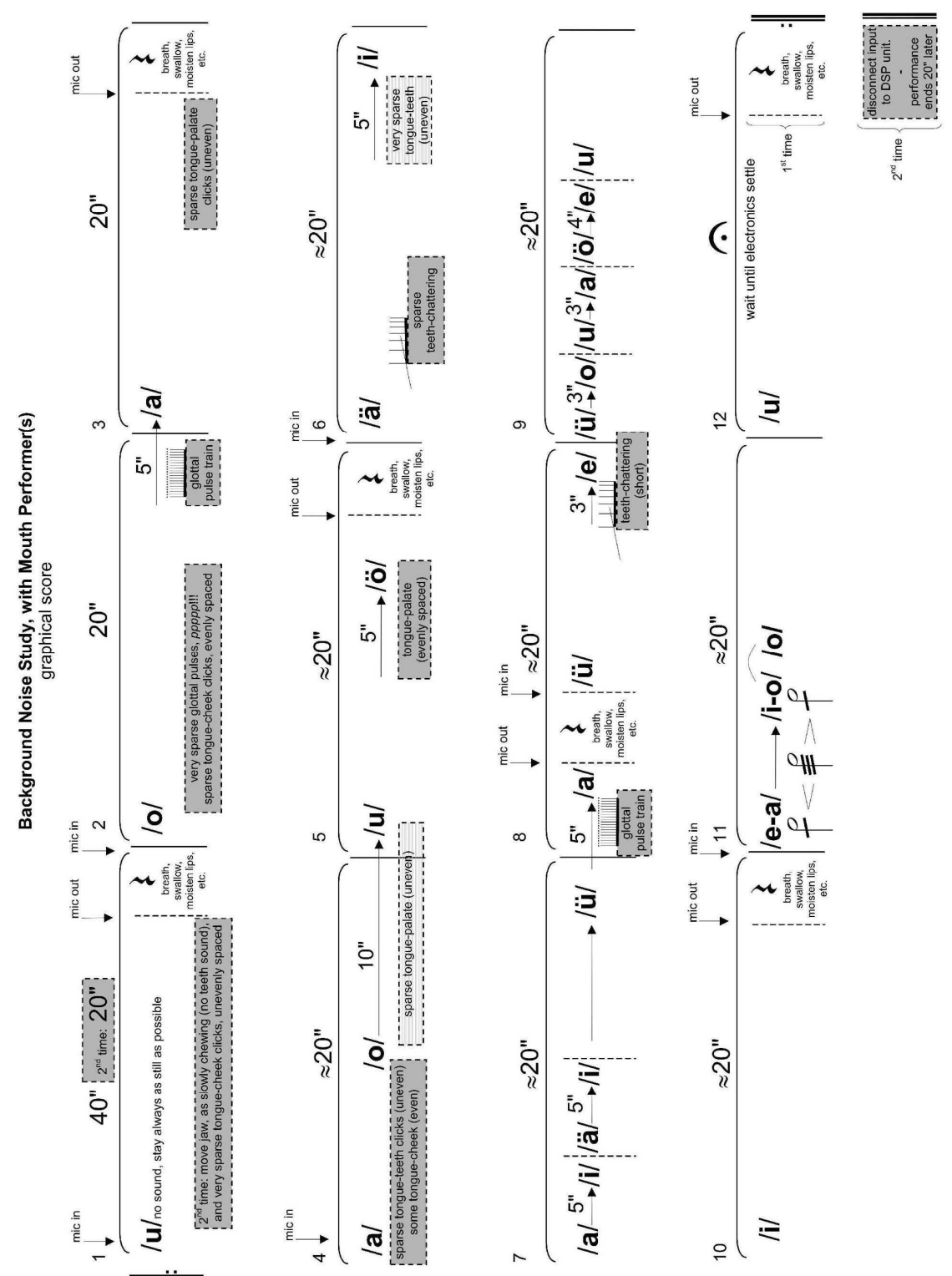

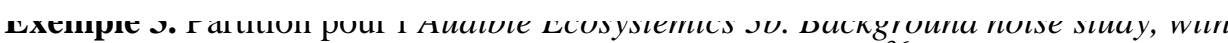
mouth performer d'Agostino Di Scipio ${ }^{36}$.

${ }^{36}$ Agostino Di SCIPIO, Audible ecosystemics, $n^{\circ} 3 a /$ Background Noise Study $n^{\circ} 3 b /$ Background Noise study, with mouth performer(s) (Instructions and score), op. cit., p 11. 
Un court échantillon extrait de cette œuvre peut être décrit comme un conglomérat de plusieurs petits sons: de nombreux grains, plusieurs impacts, des petits "clics », différents sifflements... Au-delà de cette description succincte, chaque échantillon que nous pouvons analyser est très difficile à définir et à décrire. Tous les petits sons mentionnés qu'il est possible de discerner dans un échantillon ne peuvent pas être délimités clairement dans le temps et dans l'espace. En d'autres termes, différentes échelles de temps et d'espace sont imbriquées dans un échantillon. Tous ces sons sont des mouvements multiples - innombrables - et hétérogènes : tout échantillon est un entrelacement - un réseau - complexe de mouvements disparates à des échelles différentes. Autrement dit, ces différentes échelles de temps et ces espaces variés, entremêlés dans un échantillon, vont au-delà des simples limites de ce dernier.

Au cours de la performance, les sons sont répartis sur les 8 haut-parleurs orientés vers les murs. Ainsi, tout échantillon est entendu comme un bruit de fond tout en émergeant lui-même de ce bruit de fond. Pour cette raison, il est impossible de définir une durée pour chaque mouvement imbriqué dans l'échantillon. Par exemple, nous ne pouvons pas dire quand un sifflement ou un grain apparaît et quand il disparait parce qu'il ne peut être dissocié du bruit de fond mouvant duquel il émerge. Les huit haut-parleurs tournés vers les murs créent en effet de multiples dynamiques spatiales transversales et des sons indirects: chaque événement est immergé dans un «brouhaha » dynamique tout en restant un élément actif de ce dernier. Chaque simple son n'est pas une entité indépendante (un objet): il constitue un lien spatial et temporel. Pour cette raison, chaque son ou chaque événement entendu à l'intérieur d'un échantillon analysé, peut être considéré simultanément comme un mouvement, comme une simple partie de mouvements plus amples et comme une combinaison de mouvements.

Quelles sont les racines de ces différents mouvements que nous pouvons entendre dans un échantillon? Une partie de ces derniers est directement issue du bruit de fond ambiant tout juste audible (bruits ambiants, bruits de climatisation, etc.), une autre partie provient du bruit de fond amplifié et des bruits de la cavité buccale du performer, captés 20 secondes auparavant. La dernière partie est issue des traitements effectués par le système DSP sur le bruit de fond capté. Ainsi, lorsqu'un échantillon est entendu, ces différentes parties se superposent, s'entremêlent, et s'affrontent. Tout mouvement entendu dans un échantillon peut aussi bien être une de ces parties que le résultat de la confrontation et de l'entrelacement de celles-ci.

Lorsque nous voulons analyser un échantillon pris au hasard dans l'Audible ecosystemics $3 b$, nous devons être conscient que, si certains mouvements proviennent du bruit de fond ambiant, il est impossible de retrouver cette origine et, donc, de délimiter, d'isoler, nettement chacun d'eux. Nous savons, grâce aux indications données par le compositeur, que certains mouvements sont une amplification cyclique des 20 secondes précédentes, qui sont eux-mêmes une amplification des 20 secondes précédentes, et ainsi de suite. Dans l'échantillon analysé, nous pouvons alors entendre et reconnaitre des mouvements survenus lors des 20, 40 ou 60 secondes précédentes. Certains mouvements présents - audibles dans l'échantillon 
analysé - trouvent ainsi leurs racines dans ces différents processus passés, dans ces strates spatiotemporelles variées. Dans l'espace présent (l'espace propre à l'échantillon), les espaces précédents, passés, sont «intriqués » ou «fossilisés » (à l'instar d'une empreinte). La part la plus complexe à analyser reste cependant celle issue des traitements du système DSP sur lesquels nous allons nous pencher maintenant.

Lorsque nous nous focalisons sur les traitements du système DSP, nous observons que ce que nous écoutons dans l'échantillon est spatialisé sur 8 haut-parleurs qui diffusent les signaux provenant de 7 canaux différents (cf. exemple 4 ; pour les Audible Ecosystemics. Background noise study $3 a$ et $3 b$, Agostino Di Scipio fournit les schémas du réseau des signaux de contrôle générés en direct, le schéma du réseau des signaux audio et le schéma d'assignation des haut-parleurs ${ }^{37}$ ). Deux canaux (out 1 et out 2 sur l'exemple 4) diffusent l'amplification des 20 secondes. Cinq autres canaux (out 3, 4, 5, 6, 7 sur l'exemple 4) dépendent d'un interrupteur (switch). Ce dernier est activé en fonction de l'amplitude à l'entrée du système et il contrôle plusieurs triggers qui évoluent de différentes manières. L'amplitude à l'entrée du système est dessinée par l'onde sonore erratique captée par les deux microphones : il est ainsi difficile de prévoir quand chacun de ces cinq canaux sera activé.

Ces cinq canaux instables sont issus de deux processus différents. Les trois premiers de ces 5 canaux (out 3, 4, 5 sur l'exemple 4) émettent des signaux dépendant de retards dissemblables et de triggers différemment programmés qui contrôlent chacun une vitesse de lecture du signal capté par les micros. Cela signifie que nous pouvons éventuellement reconnaître une ressemblance avec certains mouvements entendus lors des secondes précédentes. Les deux derniers canaux (out 6 et out 7 sur l'exemple 4) diffusent les signaux émis par un échantillonneur granulaire qui lit les signaux mixés provenant des trois canaux précédents. Les paramètres de cet échantillonneur granulaire (la durée de grain, la densité granulaire, le memory pointer, le memory pointer jitter) dépendent de plusieurs signaux en entrée qui évoluent selon l'amplitude du signal capté par les micros, selon les variations de l'interrupteur... Ici aussi, nous ne pouvons pas prévoir les réactions de cet échantillonneur granulaire. Tout échantillon est constitué d'une multitude de grains issus de cet échantillonneur. Or, il est impossible de saisir chacun de ces grains indépendamment l'un de l'autre; de plus, ils se combinent aux autres mouvements et les rendent instables. 


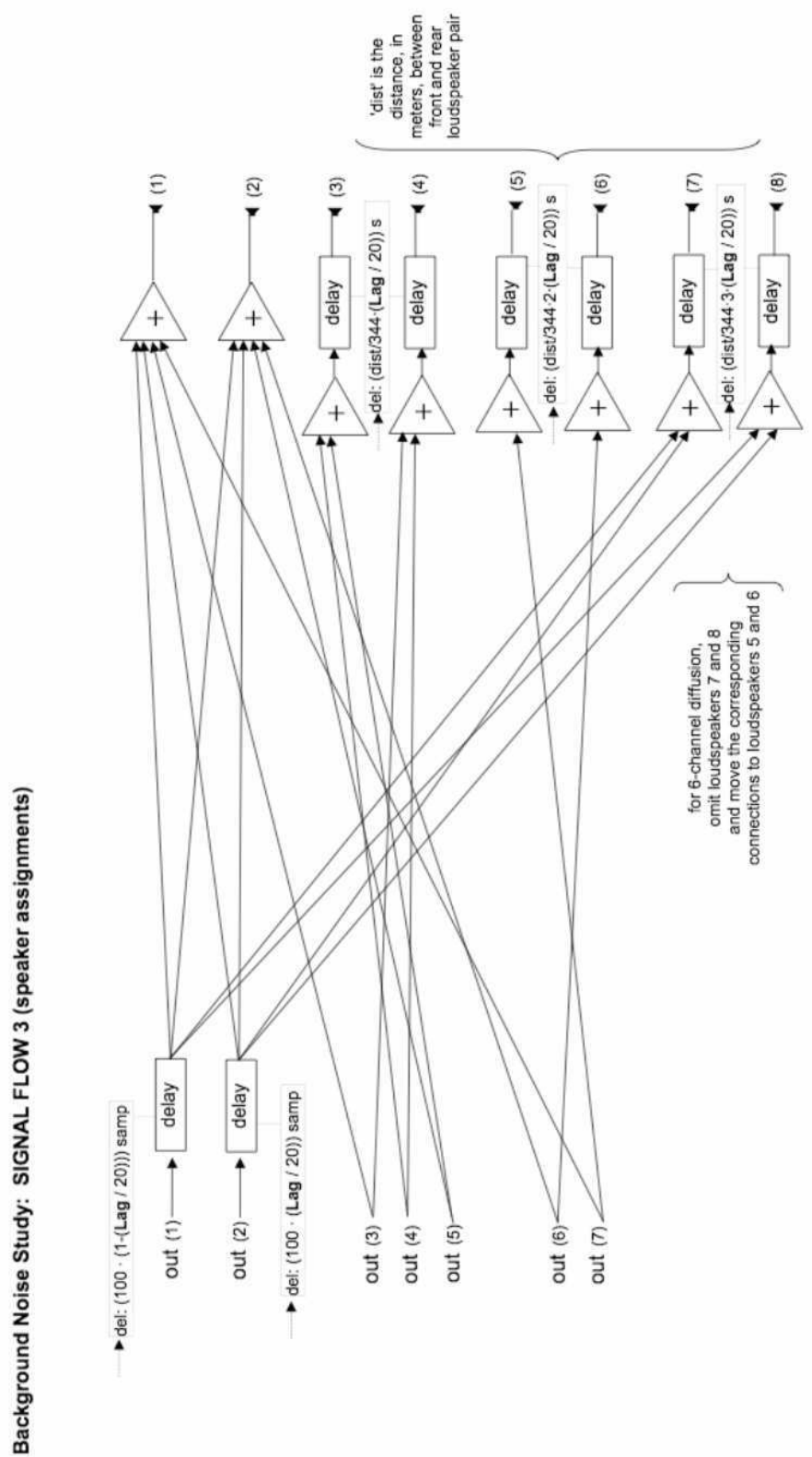

Exemple 4. Schéma d'assignation des haut-parleurs pour les Audible ecosystemics $3 a / 3 b^{38}$.

${ }^{38}$ Ibid., p. 8. 


\section{Conclusion}

Cette brève analyse - qui peut être utilisée comme base pour une analyse plus approfondie - révèle certaines caractéristiques importantes des Audible ecosystemics. Chaque mouvement - chaque échantillon - dépend d'aspects variés et il est constitué d'espaces présents et passés. Aucun échantillon ou aucun événement sonore issu de ces œuvres n'est jamais totalement achevé et bien délimité. En d'autres termes, chacun d'eux ne peut jamais être considéré comme un objet (un objet sonore) ou une image, un point qui nous fait face, dans le temps et dans l'espace. Ils se révèlent être des articulations mouvantes: une métamorphose perpétuellement en train d' ' avoir lieu » et en train de s'accomplir. Cette courte analyse tente d'appréhender cette complexité, de montrer la multiplicité des racines entrelacées dans un simple échantillon, de lier entre eux tous ces mouvements (ceux que nous pouvons entendre - les sons - et ceux qui sont inaudibles - les données DSP, les signaux numériques).

La musique d'Agostino Di Scipio - en particulier ses Audible ecosystemics constitue un exemple intéressant de structure sonore émergente. Dans ces structures spécifiques, nous ne pouvons jamais étudier indépendamment l'écoute - le traditionnel côté subjectif de la musique - et les processus informatiques - le côté objectif de la musique. Nous devons sans arrêt confronter et questionner ces deux côtés « opposés ». La notion de mouvement - ainsi que la question «qu'est-ce qui se meut dans ce que j'écoute ? »- nous permet de lier ces différents processus et de nous focaliser sur l'instabilité de ces constructions émergentes.

Confronté à la musique d'Agostino Di Scipio, celui qui analyse la musique d'une manière traditionnelle (qui isole, sépare, des entités objectives et statiques) aurait tendance à conclure qu'il s'agit d'une musique proprement inanalysable. Or, cette musique explore des technologies musicales existantes. Aujourd'hui, plus que jamais, la philosophie de la musique peut aider l'analyse musicale. 